\title{
Densidade energética da dieta e fatores associados: como está a população de São Paulo?
}

\author{
Dietary energy density and associated factors: \\ how is the population of Sao Paulo doing?
}

Dirce Maria Lobo Marchioni', Aline Mendes', Bartira Gorgulho', Roberta Horschutz Stella', Regina Mara Fisberg'

${ }^{1}$ Departamento de Nutrição em Saúde Pública, Faculdade de Saúde Pública, Universidade de São Paulo (USP), São Paulo, SP, Brasil
Correspondência para: Dirce Maria Lobo Marchioni Faculdade de Saúde Pública Universidade de São Paulo, Departamento de Nutrição

Av. Dr. Arnaldo, 715

01255-000 - São Paulo, SP, Brasil

marchioni@usp.br

Recebido em 13/Jun/2012 Aceito em 14/Ago/2012

\section{RESUMO}

Objetivo: Avaliar a densidade energética da dieta de adultos do município de São Paulo e fatores associados. Sujeitos e métodos: Participantes do estudo ISA-Capital, com amostragem probabilística ( $\mathrm{n}=710$ adultos). 0 consumo alimentar foi avaliado pelo R24h. As correlações foram investigadas pelo coeficiente de correlação de Pearson. As associações com dados demográficos, socioeconômicos e de estilo de vida foram investigadas por modelos de regressão multivariados. Resultados: A densidade energética média foi $1,98 \mathrm{kcal} / \mathrm{g}\left(\mathrm{IC}_{95 \%}[1,94 ; 2,01]\right)$ e correlacionou-se positivamente com a ingestão de energia, gordura, carboidrato, colesterol, gordura saturada, sacarose, gordura trans e açúcar adicionado e negativamente com fibras. Apenas idade e hábito de fumar apresentaram associação com a densidade energética. Conclusões: Os valores elevados da densidade energética da dieta e a relação demonstrada com outros constituintes nutricionais denotam má qualidade da dieta nessa população, o que pode estar contribuindo para crescentes taxas de excesso de peso. Arq Bras Endocrinol Metab. 2012;56(9):638-45

\section{Descritores}

Avaliação nutricional; inquérito nutricional; hábitos alimentares; ingestão de energia

\begin{abstract}
Objective: To evaluate dietary energy density in adults living in Sao Paulo and associated factors. Subjects and methods: Subjects were participants of ISA-Capital study, which was carried out with probabilistic sampling ( $\mathrm{n}=710$ adults). Food consumption was assessed by R24h. Correlations with dietary constituents were evaluated using Pearson's correlation coefficient. Associations with demographic, socioeconomic and lifestyle data were investigated by multivariate regression models. Results: The average energy density was $1.98 \mathrm{kcal} / \mathrm{g} 195 \% \mathrm{Cl}[1.94$, 2.01]). It was positively correlated with energy, fat, carbohydrate, cholesterol, saturated fat, sucrose, trans fat and added sugar intake; and negatively correlated with fiber intake. Only age and smoking were associated with energy density. Conclusions: High values of energy density and the relationship determined with other nutritional constituents denote the poor quality of the diet in this population, which may be contributing to the rising rates of overweight. Ara Bras Endocrinol Metab. 2012;56(9):638-45
\end{abstract}

Keywords

Nutrition assessment; nutrition survey; food habits; energy intake

\section{INTRODUÇÃO}

$\mathrm{O}$ $s$ índices de sobrepeso e obesidade em brasileiros apresentam-se em ascensão. Entre 2003 e 2008, o excesso de peso em adultos aumentou de $41,4 \%$ para $50,1 \%$ entre os homens e de $40,9 \%$ para $48,0 \%$ entre as mulheres (1). Já a prevalência de obesidade, nesse intervalo de cinco anos, passou de $4,1 \%$ para $5,9 \%$ entre os homens e de 3,0\% para $4,0 \%$ entre as mulheres, acompanhando a epidemia de excesso de peso observada mundialmente (1). 
Esse grave problema de saúde pública tem sido relacionado ao aumento da oferta alimentar e ao consumo crescente de alimentos com alta densidade energética (2). A densidade energética é definida como a energia disponível por unidade de peso (Quilojoules/g ou Quilocalorias/g) e relaciona-se à quantidade de água nos alimentos. Aqueles com elevada quantidade de água em sua composição, como frutas, legumes e verduras, apresentam baixa densidade energética, enquanto os com baixa quantidade de água, tais como farinhas, grãos, açúcar, óleos e manteiga, têm alta densidade energética (3).

A redução no consumo de alimentos e bebidas com alta densidade energética pode contribuir de duas formas na prevenção do ganho de peso: pela diminuição do total calórico da dieta (4) e pela regulação da ingestão de alimentos por meio do controle de sinalização do apetite (fome e saciedade) (3). Estudos controlados em laboratório mostram que os indivíduos tendem a comer, ao longo dos dias, uma quantidade constante de alimentos nas suas refeições, independente da energia fornecida por aqueles alimentos $(3,5,6)$. Dessa forma, se a densidade energética das preparações diminui, consequentemente a ingestão de energia também diminui. Por outro lado, para um mesmo valor calórico, indivíduos seguindo uma dieta de baixa densidade energética podem consumir maior quantidade de alimentos e, portanto, podem ter menor sensação de fome que indivíduos seguindo uma dieta que restringe as porções de alimentos (7).

Considerando as evidências para prevenção do ganho de peso, organizações nacionais e internacionais recomendam o consumo de alimentos de baixa densidade energética $(4,8)$. No entanto, há escassez de dados representativos da população brasileira nessa temática. Assim sendo, este trabalho visa avaliar a densidade energética da dieta de uma amostra representativa da população adulta do município de São Paulo, correlacionando-a com o valor nutritivo e com determinantes demográficos, socioeconômicos, antropométricos e de estilo de vida.

\section{CASUÍSTICA E MÉTODOS}

Foram utilizados dados da pesquisa transversal de base populacional por setores censitários "Inquéritos Alimentares no Município de São Paulo", que integra o projeto em políticas públicas "Inquérito de Saúde no Município de São Paulo - Inquérito Do- miciliar de Saúde de base populacional - ISA-Capital (2003)". As entrevistas foram conduzidas nos domicílios, durante o ano de 2003, por entrevistadores da área da saúde, previamente treinados para a aplicação dos questionários. O treinamento para realização do inquérito alimentar incluiu uma oficina conduzida em laboratório de técnica dietética para padronização de pesos e medidas de alimentos, além da simulação das entrevistas para padronização da abordagem e linguagem.

A amostra foi de 795 indivíduos adultos com idade entre 20 a 59 anos. Foram excluídos 52 indivíduos sem inquérito alimentar, 33 adultos cujo valor energético total relatado foi inferior a $500 \mathrm{kcal} /$ dia ou superior a $4.000 \mathrm{kcal} / \mathrm{dia}$, de acordo com pontos de corte propostos por Willet (9), totalizando 710 indivíduos adultos ( $47 \%$ do sexo masculino).

O consumo alimentar foi obtido por meio da aplicação do recordatório de 24 horas (R24h) em conformidade com o manual proposto por Thompson e Byers (10). O cálculo do valor nutritivo foi realizado utilizando o programa Nutrition Data System for Research (NDS-R), versão 2007 (NCC-Nutrition Cordinating Center, Minneapolis, Minnesota, USA). Esse software possui mais de 150 nutrientes, a relação de mais de 18 mil tipos de alimentos e a exportação de mais de nove tipos de arquivos de texto que permitem a análise de nutrientes, alimentos e refeições em nível individual, sendo extensivamente utilizado. Ao banco de receitas do programa, foram adicionadas preparações baseadas em receitas padronizadas $(11,12)$.

Anteriormente à digitação dos dados de consumo alimentar, as informações contidas em cada uma das coletas foram checadas a fim de monitorar a qualidade das entrevistas e definir a padronização para quantidades dos alimentos e receitas das preparações relatadas. As medidas caseiras e receitas foram compiladas de acordo com Pinheiro e cols. (11) e Fisberg e Villar (12). A seguir, os alimentos reportados pelos entrevistados foram categorizados em dois grupos: "não bebidas" $\mathrm{e}$ "bebidas".

Para a avaliação da densidade energética, consideraram-se a energia e o peso totais de alimentos ingeridos pelos indivíduos, excluindo-se as bebidas em geral $(13,14)$.

Utilizaram-se também altura e peso autorreferidos para cálculo do índice de massa corporal $\mathrm{em} \mathrm{kg} / \mathrm{m}^{2}$ (IMC). 
$\mathrm{Na}$ ausência de distribuição normal (teste ShapiroWilk), os dados foram transformados por Box-Cox a fim de viabilizar a análise paramétrica. Para averiguar a correlação entre densidade energética, energia e nutrientes, foi calculado o coeficiente de correlação de Pearson. As variáveis independentes para a análise de regressão simples foram as demográficas: idade média, sexo (masculino e feminino); socioeconômicas: situação conjugal (solteiro, unido/casado, separado/ divorciado/desquitado e viúvo), situação de trabalho (desempregado, em atividade, aposentado/pensionista e outros), escolaridade do entrevistado e chefe de família (baixa, média e alta) e renda per capita média; estilo de vida: atividade física (sedentário, insuficiente ativo, ativo e muito ativo), hábito de fumar (fumante, não fumante e ex-fumante) e consumo de bebida alcoólica (consome e não consome) e antropométricos: altura, peso e IMC (baixo peso < 18,5 $\mathrm{kg} / \mathrm{m}^{2}$, eutrofia $\geq 18,5 \mathrm{~kg} / \mathrm{m}^{2} \mathrm{e}<25 \mathrm{~kg} / \mathrm{m}^{2}$ e sobrepeso $\left.\geq 25 \mathrm{~kg} / \mathrm{m}^{2}\right)$. A atividade física foi mensurada por meio do Questionário Internacional de Atividade Física (IPAQ), versão longa, proposto pela Organização Mundial da Saúde (1998). Para calcular o gasto energético, o questionário considera informações sobre duração, frequência e intensidade das atividades. A associação linear entre essas variáveis e a densidade energética foram investigadas por meio de modelos de regressão linear, tendo a variável de interesse, densidade energética, como desfecho e as demais como variáveis explanatórias.

Em função da complexidade da amostragem, os dados foram ponderados utilizando o módulo Survey do Programa Stata versão 9 (StataCorp, Texas, USA). As análises univariadas para teste de significância das associações foram realizadas utilizando o teste $t$ de Student e o teste do Qui-quadrado para variáveis contínuas e discretas, respectivamente. Para avaliar a força da associação, foi utilizado o cálculo da razão de chances. $\mathrm{O}$ critério para seleção das variáveis para o modelo múltiplo foi a significância estatística (valor de p) menor que 0,20 na análise univariada. A estratégia de modelagem foi stepwise forward e a ordem de inserção destas foi de acordo com os valores em ordem decrescente de nível de significância. Para permanência no modelo múltiplo, foi considerado o valor de $\mathrm{p}$ menor que 0,05 ou um ajuste de, pelo menos, 10\% os coeficientes de regressão $(\beta)$ de outras variáveis já inseridas no modelo. Todas as análises de regressão foram controladas para vários potenciais fatores de confundimento. O projeto de pesquisa foi aprovado pelo Comitê de Ética da Faculdade de Saúde Pública da Universidade de São Paulo.

\section{RESULTADOS}

A idade média foi de 36,3 anos (erro-padrão [EP] $=0,48$ anos), escolaridade média de 8,1 anos ( $\mathrm{EP}=$ 0,15 anos) e do chefe da família de 7,16 anos (EP $=0,20$ ano), e a renda per capita média foi de R\$ $542,85(\mathrm{EP}=\mathrm{R} \$ 44,74)$. O IMC médio foi de 24,68 $\mathrm{kg} / \mathrm{m}^{2}\left(\mathrm{EP}=0,18 \mathrm{~kg} / \mathrm{m}^{2}\right)$. Apresentou excesso de peso (IMC $\geq 25 \mathrm{~kg} / \mathrm{m}^{2}$ ) 40,6\% da população, e 78,7\% da amostra foi considerada sedentária. Cerca de $61 \%$ eram da etnia branca. O hábito de fumar foi relatado por $22,8 \%$ dos indivíduos e o uso de bebidas alcoólicas, por $77,4 \%$. Os indivíduos com emprego formal representaram $68,3 \%$, e $60,1 \%$ estavam unidos ou casados à época da entrevista.

A média da densidade energética foi de $1,98 \mathrm{kcal} / \mathrm{g}$ $\left(\mathrm{IC}_{95 \%}[1,94 ; 2,01]\right)$. No percentil 25 , o valor da densidade energética foi de 1,68 e, no percentil 75 , foi de 2,23 . O peso médio da dieta foi de $823,45 \mathrm{~g}(\mathrm{EP}=$ $14,65 \mathrm{~g})$.

A tabela 1 apresenta a distribuição da ingestão de nutrientes, segundo tercis de densidade energética, bem como a correlação entre a densidade energética e a ingestão de energia e nutrientes. A densidade energética correlacionou-se positivamente com a ingestão de energia, gordura, carboidrato, colesterol, gordura saturada, sacarose, gordura trans e açúcar adicionado e negativamente com fibras.

Na tabela 2, observa-se que, para as médias de densidade energética, a variável hábito de fumar apresentou diferença estatisticamente significante. $\mathrm{O}$ maior valor médio de densidade energética foi observado entre indivíduos que se declararam fumantes.

Observam-se os resultados da análise de regressão linear simples e múltipla entre a densidade energética e as variáveis sociodemográficas, antropométricas e de estilo de vida na tabela 3 . Na análise múltipla, as únicas variáveis que permanecem como independentes foram $\mathrm{o}$ hábito de fumar e a idade. A associação foi inversa para idade $\left(\beta_{1}=-0,005, \mathrm{p}<0,001\right)$ e também inversa para não fumantes $\left(\beta_{1}=-0,120, \mathrm{p}<0,001\right)$ e ex-fumantes $\left(\beta_{1}=-0,184, p=0,021\right)$. O gênero e a atividade física, apesar de não significantes, permaneceram no modelo como variáveis confundidoras. 
Tabela 1. Estatística descritiva da densidade energética da dieta e da ingestão de energia e nutrientes, segundo tercis de densidade energética

\begin{tabular}{|c|c|c|c|c|c|}
\hline \multirow[b]{2}{*}{ Nutriente da dieta } & \multicolumn{3}{|c|}{ Tercis densidade energética* } & \multirow[b]{2}{*}{$r^{+}$} & \multirow[b]{2}{*}{$\mathbf{p}^{\S}$} \\
\hline & $\begin{array}{c}\text { Inferior } \\
\text { Média (EP*) }\end{array}$ & $\begin{array}{l}\text { Intermediário } \\
\text { Média }\left(\mathrm{EP}^{\star}\right)^{\dagger}\end{array}$ & $\begin{array}{c}\text { Superior } \\
\text { Média (EP*) }{ }^{\dagger}\end{array}$ & & \\
\hline Densidade energética (kcal/g) & $1,51(0,23)$ & $1,95(0,09)$ & $2,48(0,34)$ & - & - \\
\hline Energia (kcal) & $1629,93(699,46)$ & $1852,30(715,45)$ & $1958,50(763,86)$ & 0,22 & $<0,01$ \\
\hline Peso da dieta (g) & $1501,36(678,06)$ & $1431,74(611,92)$ & $1394,74(720,74)$ & $-0,05$ & 0,15 \\
\hline Proteína (g) & $68,06(34,63)$ & $76,75(34,46)$ & $70,69(32,85)$ & 0,09 & $<0,05$ \\
\hline Proteína animal (g) & $38,47(29,38)$ & $46,86(29,00)$ & $44,30(29,14)$ & 0,03 & 0,36 \\
\hline Proteína vegetal (g) & $29,37(16,87)$ & $29,62(14,32)$ & $25,89(12,85)$ & 0,07 & 0,06 \\
\hline Gordura (g) & $52,80(26,09)$ & $71,13(31,55)$ & $82,35(37,63)$ & $-0,07$ & 0,06 \\
\hline Carboidrato (g) & $217,24(99,32)$ & $221,13(92,56)$ & $227,20(97,48)$ & 0,38 & $<0,01$ \\
\hline Colesterol (mg) & $176,87(139,68)$ & $205,70(135,71)$ & $210,57(159,90)$ & 0,38 & $<0,01$ \\
\hline Gordura saturada (g) & $16,07(10,28)$ & $21,73(10,97)$ & $26,92(13,39)$ & 0,41 & $<0,01$ \\
\hline Sacarose (g) & $29,22(30,67)$ & $27,97(24,86)$ & $40,85(39,16)$ & 0,09 & $<0,05$ \\
\hline Fibras (g) & $17,90(9,33)$ & $15,94(8,87)$ & $13,36(7,60)$ & $-0,22$ & $<0,01$ \\
\hline Cálcio (mg) & $553,42(374,51)$ & $530,00(324,59)$ & $568,81(1679,97)$ & 0,04 & 0,23 \\
\hline Sódio (mg) & $2985,16(1646,72)$ & $3139,95(1333,05)$ & $3136,75(1679,97)$ & 0,05 & 0,21 \\
\hline Gordura trans (g) & $2,66(2,27)$ & $4,23(3,24)$ & $6,55(5,07)$ & 0,22 & $<0,01$ \\
\hline Açúcar adicionado (g) & $33,02(38,88)$ & $38,28(36,56)$ & $57,87(53,42)$ & 0,31 & $<0,01$ \\
\hline
\end{tabular}

*Valores ponderados conforme desenho amostral; † EP: erro-padrão; ${ }^{\circ}$ Coeficiente de correlação de Pearson; ${ }^{\S}$ Valor de $p$.

Tabela 2. Análise descritiva da densidade energética da dieta, segundo variáveis sociodemográficas, nutricionais e de estilo de vida

\begin{tabular}{|c|c|c|c|c|}
\hline Variável & Característica & Média & EP* & $\mathbf{p}^{\dagger}$ \\
\hline \multirow[t]{2}{*}{ Gênero } & Masculino & 1,96 & 0,024 & 0,358 \\
\hline & Feminino & 1,99 & 0,025 & \\
\hline \multirow[t]{5}{*}{ Etnia $^{\ddagger}$} & Branca & 1,94 & 0,026 & 0,174 \\
\hline & Negra & 2,00 & 0,064 & \\
\hline & Parda & 2,04 & 0,035 & \\
\hline & Amarela & 1,82 & 0,142 & \\
\hline & Indígena & 1,92 & 0,000 & \\
\hline \multirow[t]{3}{*}{ Índice de massa corporal\$ } & Menor que 18,5 kg/m² & 1,93 & 0,079 & 0,368 \\
\hline & 18,5 a $24,9 \mathrm{~kg} / \mathrm{m}^{2}$ & 1,99 & 0,025 & \\
\hline & Maior ou igual que $25,0 \mathrm{~kg} / \mathrm{m}^{2}$ & 1,97 & 0,028 & \\
\hline \multirow[t]{3}{*}{ Hábito de fumar/" } & Não fumante & 1,98 & 0,024 & 0,007 \\
\hline & Ex-fumante & 1,88 & 0,046 & \\
\hline & Fumante & 2,02 & 0,041 & \\
\hline \multirow[t]{2}{*}{ Consumo de bebida alcoólica" } & Não consome & 2,02 & 0,040 & 0,391 \\
\hline & Consome & 1,97 & 0,019 & \\
\hline \multirow[t]{4}{*}{ Situação de trabalho** } & Desempregado & 2,02 & 0,051 & 0,299 \\
\hline & Em atividade & 1,99 & 0,025 & \\
\hline & Aposentado ou pensionista & 1,91 & 0,072 & \\
\hline & Outros (dona de casa, estudante, outros) & 1,92 & 0,036 & \\
\hline \multirow[t]{4}{*}{ Situação conjugal ${ }^{\dagger \dagger}$} & Solteiro & 2,01 & 0,036 & 0,167 \\
\hline & Unido/casado & 1,96 & 0,026 & \\
\hline & Separado/divorciado/desquitado & 2,04 & 0,066 & \\
\hline & Viúvo & 1,89 & 0,069 & \\
\hline \multirow[t]{4}{*}{ Atividade física ${ }^{\ddagger \ddagger}$} & Sedentário & 1,98 & 0,022 & 0,249 \\
\hline & Insuficiente ativo & 2,07 & 0,069 & \\
\hline & Ativo & 1,98 & 0,082 & \\
\hline & Muito ativo & 1,91 & 0,062 & \\
\hline
\end{tabular}

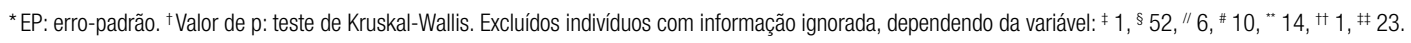


Tabela 3. Análise de regressão linear simples e múltipla: beta e intervalo de confiança de 95\% (IC 95\%) entre a DE variáveis sociodemográficas, nutricionais e de estilo de vida

\begin{tabular}{|c|c|c|c|c|c|}
\hline Variável independente & Categorias & $\beta 1^{*},+$ & $\mathbf{p}^{\star, \S}$ & $\beta 1^{t, \pm}$ & $\mathbf{p}^{\mathbf{t}, \S}$ \\
\hline \multirow[t]{2}{*}{ Gênero } & Masculino & & & & \\
\hline & Feminino & 0,018 & 0,475 & 0,02 & NS \\
\hline Idade (anos) & - & $-0,006$ & 0,000 & $-0,005$ & $<0,001$ \\
\hline \multirow{2}{*}{$\begin{array}{l}\text { Consumo de bebida } \\
\text { alcoólica }\end{array}$} & Não consome & & & & \\
\hline & Consome & $-0,030$ & 0,300 & - & - \\
\hline \multirow[t]{3}{*}{ Hábito de fumar } & Fumante & & & & \\
\hline & Não fumante & $-0,060$ & 0,050 & $-0,120$ & $<0,001$ \\
\hline & Ex-fumante & $-1,00$ & 0,013 & $-0,184$ & 0,021 \\
\hline \multirow[t]{2}{*}{ Situação do trabalho } & Desempregado & & & & \\
\hline & Em atividade & $-0,036$ & 0,386 & - & - \\
\hline \multirow[t]{2}{*}{ Índice de massa corporal } & Adequado & & & & \\
\hline & Excesso de peso & $-0,023$ & 0,390 & - & - \\
\hline \multirow[t]{2}{*}{ Situação conjugal } & Solteiro & & & & \\
\hline & Unido/casado & $-0,039$ & 0,118 & - & - \\
\hline \multirow[t]{3}{*}{ Escolaridade do indivíduo } & Baixa & & & & \\
\hline & Média & 0,031 & 0,263 & - & - \\
\hline & Alta & $-0,013$ & 0,705 & - & - \\
\hline \multirow[t]{4}{*}{ Atividade física } & Sedentário & & & & \\
\hline & Insuficiente ativo & 0,068 & 0,189 & 0,068 & NS \\
\hline & Ativo & 0,010 & 0,847 & $-0,003$ & NS \\
\hline & Muito ativo & $-0,057$ & 0,193 & $-0,065$ & NS \\
\hline
\end{tabular}

${ }^{\star}$ Regressão linear simples; ${ }^{\dagger}$ regressão linear múltipla; ${ }^{\sharp} \beta$ : Coeficiente de regressão; ${ }^{\S} \mathrm{p}$ : valor de $p$.

\section{DISCUSSÃO}

No presente estudo, conduzido em amostra representativa do Município de São Paulo, foram observados valores elevados de densidade energética da dieta, fato que pode estar contribuindo para as crescentes taxas de sobrepeso e obesidade relatadas nessa população. A média de densidade energética observada, $1,98 \mathrm{kcal} / \mathrm{g}$, foi superior ao valor recomendado (menor que 1,25 $\mathrm{kcal} / \mathrm{g}$ ) (15). Chama a atenção que o valor médio observado no tercil inferior da densidade energética, 1,51 $\mathrm{kcal} / \mathrm{g}$, já supera esse valor. No entanto, nossos resultados estão em concordância com outros descritos na literatura, demonstrando uma tendência atual de consumo de alimentos com maior densidade energética $(2,14,16)$.

A densidade energética média encontrada nos participantes do Continuing Survey of Food Intakes by Individuals (CSFII) de 1994 a 1996 e em indivíduos do terceiro National Health and Nutrition Examination Survey (NHANES III) de 1999 a 2004 foi de 1,85 e $1,97 \mathrm{kcal} / \mathrm{g}$, respectivamente $(14,16)$. Para adultos maiores de 19 anos do National Health and Nutrition
Examination Surveys (2003-06), nos Estados Unidos, os valores foram ainda maiores, de $2,05 \mathrm{kcal} / \mathrm{g}(2)$. Esses achados são preocupantes, tendo em vista o crescente corpo de evidências que relaciona a densidade energética da dieta ao peso corporal em adultos (17).

É reconhecido que indivíduos que consomem uma maior quantidade de energia estão mais sujeitos a alcançar as recomendações para nutrientes, pela elevada correlação entre energia e nutrientes (18). Assim, teoricamente, restringir a energia poderia levar ao comprometimento da ingestão de nutrientes. No entanto, a densidade energética correlacionou-se diretamente com calorias, gordura, colesterol, gordura saturada e gordura trans e inversamente com fibras. A fibra é um nutriente-chave para a redução da densidade energética, uma vez que a contribuição para o valor energético é pequena em comparação à sua contribuição para o peso da refeição (19). Estudos anteriores descreveram associação similar com gordura e fibras $(16,20)$.

Nosso estudo acrescenta evidências a outros estudos anteriores $(21,22)$ mostrando que uma dieta com baixa densidade energética leva a menor ingestão de energia, 
apesar do consumo de maior quantidade de alimentos. O menor peso da dieta no tercil superior da densidade energética, relatado no presente estudo, indica que o aumento da densidade energética pode ser atribuído à ingestão de alimentos com menor quantidade de água e maior quantidade de calorias por unidade de peso, como alimentos ricos em gordura, ou preparações com sacarose e açúcar de adição. Esse achado está em conformidade com estudos anteriores que relacionam dietas com menor densidade energética com dietas de maior qualidade $(23,24)$.

As evidências mostram que uma dieta com baixa densidade energética é uma dieta saudável, baseada em alimentos de origem vegetal e que inclui alimentos de origem animal, como carnes magras e laticínios com teor de gordura reduzido $(21,23,24)$. As frutas e hortaliças, cujo consumo é estratégico para baixar a densidade energética da dieta, associam-se à redução de fatores de risco para doenças cardiovasculares, prevenção do câncer e decréscimo de mortalidade por todas as causas (25). No entanto, de acordo com estudos anteriores realizados nessa mesma população, o consumo é baixo, muito aquém do mínimo recomendado de $400 \mathrm{~g} /$ dia (26). Apenas $6,5 \%$ dos adolescentes consumiam frutas e hortaliças adequadamente e $22 \%$ não consumiam nenhum tipo (26) e, entre os adultos, $50 \%$ não consumiram nenhuma porção de fruta no dia investigado (27). A inadequação da ingestão também é confirmada pela Pesquisa de Orçamento Familiar (POF) (2008-2009), que analisou 55.970 domicílios brasileiros e mostrou que as frutas e hortaliças correspondiam a apenas 2,8\% da aquisição alimentar do brasileiro, sendo o ideal estimado entre $9 \%$ e $12 \%$, com base em uma dieta de $2.000 \mathrm{kcal} / \mathrm{pessoa} /$ dia e na recomendação de $400 \mathrm{~g} /$ dia (28). Também na Europa e nos Estados Unidos é relatado o baixo consumo $(21,29)$.

Além disso, esse padrão dietético tem sido descrito tanto em estudos " $a$ priori", por técnicas estatísticas como análise fatorial, quanto "a posteriori", por índices de qualidade da dieta. No entanto, como padrões de consumo são culturalmente determinados, o uso desses métodos torna difíceis as comparações entre estudos em populações distintas e ao longo do tempo. Nesse sentido, a medida da densidade energética da dieta poderia superar essa dificuldade, além de ser mais fácil de calcular $(21,30)$.

Dos fatores sociais, demográficos, de estilo de vida e antropométricos investigados, apenas a idade e o hábito de fumar associaram-se, negativamente, à densidade energética. Pesquisa anterior, utilizando a mesma população deste estudo, relata associação negativa entre qualidade da dieta e idade, consistente com os dados da presente pesquisa (27). Em relação ao hábito de fumar, outros estudos mostraram que fumantes têm ingestão mais baixa de fibra, vitamina $C$, vitamina $E$ e betacaroteno, nutrientes correlacionados com a ingestão de frutas e vegetais, ao lado de maior consumo de álcool e energia $(31,32)$.

Porém, destaca-se que, em todas as categorias das variáveis analisadas, a densidade energética foi elevada: o menor valor médio foi de $1,82 \mathrm{kcal} / \mathrm{g}$. As escolhas alimentares são baseadas no gosto (paladar), no custo e na conveniência (33). O gosto, uma resposta hedônica ao alimento, é largamente dependente da densidade energética. Os alimentos mais palatáveis em geral são aqueles que concentram a maior energia por unidade de volume. Quanto ao custo, dietas de baixa densidade energética, ricas em frutas e vegetais, são em geral mais caras (34). Quanto à conveniência, produtos frescos costumam ser mais difíceis de transportar, preparar e estocar.

Entre as limitações deste estudo, podemos citar a análise de um único dia da dieta, não caracterizando, portanto, uma análise da densidade energética baseada no consumo alimentar habitual (35). Porém, o consumo médio usual de um grupo de indivíduos pode ser obtido se forem contemplados todas as estações do ano e todos os dias da semana durante a aplicação do inquérito $(24,36)$, o que ocorreu no estudo ISA-Capital 2003. O uso de medidas antropométricas relatadas e não aferidas pode resultar em erro de classificação e atenuar a relação entre os dois fenômenos analisados. Porém, estudos realizados em populações brasileiras demonstram alta correlação, sensibilidade e especificidade dessas medidas quando pareadas com as aferidas $(37,38)$. Além disso, apesar de não haver consenso sobre o método a ser usado para o cálculo da densidade energética, duas recentes revisões sistemáticas apoiam a exclusão das bebidas, e a maioria dos estudos vem adotando essa abordagem, que também foi adotada no nosso estudo $(17,39)$. Considerando que os alimentos e as bebidas têm efeitos diferentes na saciedade e na ingestão energética, esses dois fatores dietéticos devem ser tratados separadamente. A recomendação para realizar o cálculo da densidade energética sem considerar as bebidas não significa ignorar seu papel na rede causal da obesidade. Nesse sentido, recomenda-se que o consumo de bebidas seja utilizado como covariável na investigação da relação obesidade e densidade energé- 
tica. Finalmente, o desenho do estudo, transversal, não permite supor relações de causalidade.

Os dados deste estudo demonstram que a densidade energética da dieta dos residentes de São Paulo é elevada, superando o preconizado já no percentil 25. Além disso, a inexistente relação da densidade energética com a maioria dos fatores sociais, demográficos e de estilo de vida investigados mostra a generalização desse fenômeno. Nosso estudo traz evidências da urgente necessidade de ações para melhoria da qualidade da dieta dessa população, e nossos dados podem apoiar o delineamento de ações programáticas para o consumo de dietas de menor densidade energética, que podem ser consideradas uma estratégia para prevenção do ganho de peso. No entanto, mais estudos são necessários para elucidar as relações já demonstradas em outros países na nossa população.

Agradecimentos: ao apoio financeiro do Conselho Nacional de Desenvolvimento Científico e Tecnológico $(\mathrm{CNPq})$ para a realização desta pesquisa.

Declaração: os autores declaram não haver conflitos de interesse científico neste estudo.

\section{REFERÊNCIAS}

1. IBGE - Instituto Brasileiro de Geografia e Estatística. Programa de Orçamentos Familiares (2002-2003) - Análise da Disponibilidade Domiciliar de Alimentos e do Estado Nutricional no Brasil. Rio de Janeiro; 2003.

2. Duffey KJ, Popkin BM. Energy density, portion size, and eating occasions: contributions to increased energy intake in the United States, 1977-2006. PloS Med. 2011;8(6):e1001050.

3. Rolls BJ, Roe LS, Meengs JS. Reductions in portion size and energy density of foods are additive and lead to sustained decreases in energy intake. Am J Clin Nutr. 2006;83(1):11-7.

4. WHO, FAO. Diet, nutrition and the prevention of chronic diseases. WHOTechnical Report Series 916. Genebra; 2003.

5. Song SW, Bae YJ, Lee DT. Effects of caloric restriction with varying energy density and aerobic exercise on weight change and satiety in young female adults. Nutr Res Pract. 2010;4(5):414-20.

6. Blatt $A D$, Roe LS, Rolls BJ. Hidden vegetables: an effective strategy to reduce energy intake and increase vegetable intake in adults. Am J Clin Nutr. 2011;93(4):756-63.

7. Ello-Martin JA, Roe LS, Ledikwe JH, Beach AM, Rolls BJ. Dietary energy density in the treatment of obesity: a year-long trial comparing 2 weight-loss diets. Am J Clin Nutr. 2007;85(6):1465-77.

8. Ministério da Saúde. Guia Alimentar para a População Brasileira: promovendo a alimentação saudável. Série A. Brasília; 2005.

9. Willet WC. Nutritional Epidemiology. 2.ed. NewYork (NY): Oxford Press; 1998.

10. Thompson FE, Byers T. Dietary assessment resource manual. J Nutr. 1994;124(11S):2245S-2317S.

11. Pinheiro AB, Lacerda EM, Benzecry EH, Gomes MCS, Costa VM. Tabela para avaliação de consumo alimentar em medidas caseiras. 2.ed. Rio de Janeiro: UERJ; 1994.
12. Fisberg RM, Villar BS. Manual de Receitas e Medidas Caseiras para Cálculo de Inquéritos Alimentares. São Paulo: Signus; 2002.

13. Cox DN, Mela DJ. Determination of energy density of freely selected diets: methodological issues and implications. Int J Obes. 2000;24(1):49-54.

14. Ledikwe JH, Blanck HM, Khan LK, Serdula MK, Seymour JD, Tohill $\mathrm{BC}$, et al. Dietary energy density determined by eight calculation methods in a nationally representative United States population. J Nutr. 2005;135(2):273-8.

15. World Cancer Research Fund/American Institute for Cancer Research. Food, Nutrition, Physical Activity and the Prevention of Cancer: a Global Perspective. AICR: Washington; 2007.

16. Kant AK, Andon MB, Angelopoulos TJ, Rippe JM. Association of breakfast energy density with diet quality and body mass index in American adults: National Health and Nutrition. Am J Clin Nutr. 2008;88(5):1999-2004.

17. Pérez-Escamilla R, Obbagy JE, Altman JM, Essery EV, Mcgrane MM, Wong YP, et al. Dietary energy density and body weight in adults and children: a systematic review. J Acad Nutr Diet. 2012;112(5):671-84.

18. Newby P. Examining energy density: comments on diet quality, dietary advice, and the cost of healthful eating. J Am Diet Assoc. 2006;106(8):1166-9.

19. Rolls BJ, Drewnowski A, Ledikwe JH. Changing the energy density of the diet as a strategy for weight management. J Am Diet Assoc. 2005;105(5S1):S98-103.

20. Raynor HA, Van Walleghen EL, Bachman JL, Looney SM, Phelan S, Wing RR. Dietary energy density and successful weight loss maintenance. Eat Behav. 2011;12(2):119-25.

21. Ledikwe JH, Blanck HM, Kettel Khan L, Serdula MK, Seymour JD, Tohill BC, et al. Dietary energy density is associated with energy intake and weight status in US adults. Am J Clin Nutr. 2006;83(6):1362-8.

22. Piernas $C$, Popkin BM. Increased portion sizes from energy-dense foods affect total energy intake at eating occasions in US children and adolescents: patterns and trends by age group and sociodemographic characteristics. Am J Clin Nutr. 2011;94(5):1324-32.

23. Esmaillzadeh A, Azadbakht L. Dietary energy density and the metabolic syndrome among Iranian women. Eur J Clin Nutr. 2011;65(5):598-605.

24. Previdelli AN, Andrade SCD, Pires MM, Ferreira SRG, Fisberg RM, Marchioni DM. A revised version of the Healthy Eating Index for the Brazilian population. Rev Saúde Pública. 2011;45(4):794-8.

25. US Department of Health and Human Services. Tracking Healthy People 2020: nutrition and weight status [Internet]. Washington; 2012. Disponível em: http://www.healthypeople.gov/2020/topicsobjectives2020/overview.aspx?topicid=29. Acesso em: $20 \mathrm{Jul}$, 2012.

26. Bigio RS, Verly Junior E, Castro MAD, César CLG, Fisberg RM, Marchioni DML. Determinantes do consumo de frutas e hortaliças em adolescentes por regressão quantílica. Rev Saúde Pública. $2011 ; 45(3): 448-56$.

27. Morimoto JM, Latorre $\mathrm{M}$ do $\mathrm{R}$, César $\mathrm{CL}$, Carandina L, Barros $M B$, Goldbaum MFR. Fatores associados à qualidade da dieta de adultos residentes na região metropolitana de São Paulo, Brasil, 2002. Cad Saúde Pública. 2008;24(1):169-78.

28. Bertazzi LR, Moreira CR, Lenise M, Rosely S, Augusto MC. Distribuição regional e socioeconômica da disponibilidade domiciliar de alimentos no Brasil em 2008-2009. Rev Saúde Pública. 2012;46(1):6-15.

29. Drewnowski A, Darmon N.The economics of obesity: dietary energy density and energy cost. Am J Clin Nutr. 2005;82(1S):265S$-73 S$.

30. Patterson E, Wärnberg J, Poortvliet E, Kearney JM, Sjöström M. Dietary energy density as a marker of dietary quality in Swedish 
children and adolescents: the European Youth Heart Study. Eur J Clin Nutr. 2010;64(4):356-63.

31. Dallongeville J, Marecaux N, Fruchart JC, Amouye P. Cigarrette smoking is associated with unhealthy patterns of nutrient intake: a meta-analysis. J Nutr. 1998;128:1450-7.

32. Alberg $A$. The influence of cigarette smoking on circulating concentrations of antioxidant micronutrients. Toxicology. 2002;180(2):121-37.

33. Eikenberry N, Smith C. Healthful eating: perceptions, motivations, barriers, and promoters in low-income Minnesota communities. J Am Diet Assoc. 2004;104:1158-61.

34. Connell CL, Zoellner JM, Yadrick MK, Chekuri SC, Crook LB, Bogle ML. Energy density, nutrient adequacy, and cost per serving can provide insight into food choices in the lower Mississippi Delta. J Nutr Educ Behav. 2011;44(2):148-53.

35. Dodd KW, Guenther PM, Freedman IS, Subar AF, Kipnis V, Midthune $D$, et al. Statistical methods for estimating usual intake of nutrients and foods: a review of the theory. J Am Diet Assoc. 2006;106:1640-50.

36. Miller PE, Mitchell DC, Harala PL, Pettit JM, Smiciklas-Wright $H$, Hartman TJ. Development and evaluation of a method for calculating the Healthy Eating Index-2005 using the Nutrition Data System for Research. Public Health Nutr. 2011;14(2):306-13.

37. Lucca A, Moura EC. Validade e confiabilidade do peso, estatura e índice de massa corporal auto-referidos obtidos por entrevista telefônica. Cad Saúde Pública. 2010;26(1):110-22.

38. Fonseca M de J, Faerstein E, Chor D, Lopes CS. Validade de peso e estatura informados e índice de massa corporal: estudo pró-saúde. Rev Saúde Pública. 2004;38(3):392-8.

39. Johnson L, Wilks DC, Lindroos AK, Jebb SA. Reflections from a systematic review of dietary energy density and weight gain: is the inclusion of drinks valid?. Obes Rev. 2009;10(6):681-92. 